\title{
Aromatic Polymers Obtained by Precipitation Polycondensation. 3. Thermal Behavior and Microstructure of PEKEKK Particles
}

\author{
D. R. Rueda, M. G. Zolotukhin, M. E. Cagiao, and F. J . Balta Calleja* \\ Instituto de Estructura de la Materia, C.S.I.C., Serrano 119, Madrid 28006, Spain
}

\section{Villers and M. Dosière}

Université de Mons-Hainaut, Phys.-Chem. des Polymeres, Place du Parc 20, B-3000 Mons, Belgium

Received March 29, 1996; Revised Manuscript Received J uly 30, $1996^{\otimes}$

\begin{abstract}
In the second part of this series the influence of monomer concentration on the chemical structure and the shape and size of poly(aryl ether ketone ether ketone ketone) (PEKEKK) particles was reported. The present study of the thermal behavior and microstructure of aromatic polyketone samples in the form of particles has been carried out by means of differential scanning calorimetry (DSC) and wide angle X-ray diffraction (WAXD). Samples before and after reprecipitation and annealing were investigated. Similar to other polyketones, PEKEKK shows two polymorphic forms: form II, less stable, is currently observed in the samples, while the reprecipitated polymer preferentially shows form I. Form II is partially converted into form I upon short annealing treatment of the material. The semicrystalline materials show melting points and glass transition temperatures which are higher for the samples with higher molecular weight and more regular chain structure. A comparison of thermal properties of "as obtained" and "reprecipitated" samples reveals the influence of the particle architecture on the chain mobility. The latter determines the ability of polyketone chains to crystallize. Infrared dichroism was also used to characterize the arrangement of polymer chains inside the polyketone particles showing crystal habits.
\end{abstract}

\section{Introduction}

Aromatic poly(ether ketone)s as high-performance thermoplastic materials have received special attention in the last decade. ${ }^{1-3}$ These polymers show high thermostability and resistance to chemical agents 2,3 and are usually semicrystalline. A basicinterest for polyketones was also prompted by the fact that polyketones show a double melting behavior ${ }^{4-6}$ when crystallized at temperatures above the glass transition temperature. The origin of this phenomenon is still open to discussion.

With reference to the structure physical properties correlation it is known that the thermal properties (melting and glass transition temperatures) of paralinked aromatic polyketones are very dependent on the ether/ketone ratio which influences the lattice parameters of the orthorhombic unit cell. The chain packing is similar to that found for poly(phenylene oxide) ${ }^{7}$ and for PEEK ${ }^{8-10}$ For other aromatic polyketones the $b$ and clattice parameters increase linearly with the carbonyl content while the a parameter decreases. For polyketones with a carbonyl content larger than 50\% (PEKEKK and PEKK) Blundell and Newton ${ }^{11}$ observed additional reflections in the X-ray pattern of oriented samples which were indexed in terms of a new orthorhombic crystallographic form with the $a$ and $b$ axes exchanged with each other.

Gardner et al. reported new results on the polymorphism of aromatic polyketones with different ether/ ketone ratios ${ }^{12,13}$ crystallized under different conditions, and more recently for polyketones containing isophthalic

\footnotetext{
* To whom correspondence is addressed.

† Part 2: M. G. Zolotukhin et al. (submitted for publication to Macromol. Chem., Phys.).

${ }_{\otimes}$ Abstract published in AdvanceACS Abstracts, September 15, 1996.
}

units. ${ }^{14}$ In the case of polyketones crystallized from the melt the first orthorhombic packing (PEEK-type) is always observed for all polyketones independently of their carbonyl content. On the other hand, samples prepared by cold crystallization (from amorphous mate rial upon thermal treatement) and solvent crystallization, with a carbonyl content larger than 50\% (PEKEKK, PEKK), showed exclusively the second crystallographic form. For the polyketone PEK the first crystal lographic form is still predominant in relation to the second one. In this paper we will refer to these polymorphic forms as form I and form II following Gardner's notation.

It is assumed that aromatic polyketones are linear, with para substitution in arylene rings of the main chain. Nevertheless, regardless of the preparation method (nucleophilic or electrophilic routes) defect isomeric structures can be present along the linear polyketone chains. The presence of these defect structures, even in small relative amounts can obviously affect the chain packing and relative motion of chains and, consequently, the microstructure and thermal behavior of the material. In the second paper of this series $^{15}$ the preparation and NMR characterization of the aromatic polyketones investigated here were reported. Such a detailed chemical characterization confirmed a good quality and homogeneity of the materials, and it encouraged us to investigate the variation of the thermal properties and the microstructure as a function of (a) the mol ecular weight and chain regularity and (b) the architecture of the particles obtained. Thus, a characterization of the polyketone samples by means of X-ray diffraction and differential scanning calorimetry is presented. I nfrared dichroism was also used for a better characterization of the building up of polyketone particles which showed a needl elike crystal habit. 
Table 1

\begin{tabular}{ccc}
\hline & viscosity & $\begin{array}{c}\text { defect isomer } \\
\text { molar content }(\%)\end{array}$ \\
\hline P1 & 1.46 & 6.7 \\
P2 & 1.85 & 5.0 \\
P3 & 2.42 & 2.4
\end{tabular}

\section{Experimental Part}

Materials. The chemical repeat unit of poly(aryl ether ketone ether ketone ketone) is

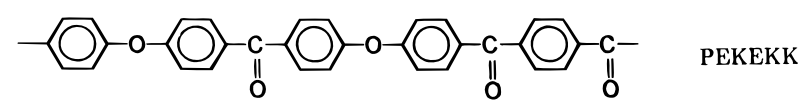

Three PEKEKK samples prepared by the precipitation polycondensation method were investigated. The samples obtained in particle form show different molecular weights (viscosity) and different isomer unit contents (Table 1). The isomer unit content refers to the relative total amount of 1,2 and 1,3 substituted phenylene rings. A description of the preparation and characterization of samples is given elsewhere. ${ }^{15}$

Let us remember here that both the shape and the size of the particles were found to be governed by reaction conditions. The P1 sample consists of smooth, elongated particles (0.4$0.7 \mathrm{~mm}$ ). The P2 sample consists of spherical particles of about $0.1 \mathrm{~mm}$ diameter in the form of el ongated aggregates $(<1 \mathrm{~mm}$ long). The $\mathrm{P} 3$ sample in addition to the aggregates of spherical particles shows very thin needlelike crystals.

Methods. For the thermal study a differential scanning calorimeter Perkin-Elmer DSC-4 was employed. A heating rate of $20 \mathrm{~K} / \mathrm{min}$ was used. After the first DSC run the sample was rapidly cooled to $90{ }^{\circ} \mathrm{C}$ and then a second DSC run was made at the same heating rate. DSC pans, carefully filled with polymer particles, contained about 5-8 $\mathrm{mg}$ of material.

To examine the role of the architecture of the particles themsel ves on the properties, the materials were dissolved in a $\mathrm{CF}_{3} \mathrm{COOH}-\mathrm{CDCl}_{3}$ mixture and reprecipitated using methanol.

A vertical powder diffractometer (Rigaku) was used to obtain wide angle X-ray diffractograms (WAXD) using a $\mathrm{Cu} \mathrm{K} \alpha, \mathrm{Ni}$ filtered radiation source from a rotating anode working at 8 $\mathrm{kW}$. A scanning speed of $1^{\circ}(2 \theta)$ per min was used. To overcome the difficulties in filling the sample holder with the same amount of the particle sample, we have prepared sintered disks ( $\phi=13 \mathrm{~mm}$ ) using $100 \mathrm{mg}$ of material. For sintering, the samples were evacuated for $5 \mathrm{~min}$ followed by application of a pressure of $0.75 \mathrm{MPa}$ under vacuum for another $5 \mathrm{~min}$. Thus, X-ray diffratograms from sintered samples are more comparable and reliable than those directely obtained from particle samples. The crystallinity of the samples was calculated as the ratio of the crystalline area to the total diffracted area after subtraction of a linear background within the angular region between 5 and $36^{\circ}(2 \theta)$. To separate both the crystalline and amorphous contribution from the experimental scattering pattern, we have considered an amorphous halo centered at $20^{\circ}(2 \theta)$ which tangentially approaches the experimental curve at $2 \theta$ values of 14,26 , and $35^{\circ}$.

In order to promote crystallinity, all the sintered samples and the reprecipitated material were annealed at $230{ }^{\circ} \mathrm{C}$ for 15 min. Particularly, sample P 3 was thermally treated up to temperatures closer to the melting point to study the polymorphism.

A flat camera was also used to obtain X-ray patterns of the particle samples, which were inserted in a glass capillary ( 2 and $0.7 \mathrm{~mm}$ diameter). A distance from the sample to X-ray film of $40 \mathrm{~mm}$ was used.

Polarized infrared spectra of P3 needlelike particles were obtained on a Bruker IFS 113V FTIR spectrometer using an aluminum wire grid polarizer. The spectra were obtained after 32 accumulated scans with a resolution of $2 \mathrm{~cm}^{-1}$. The longest

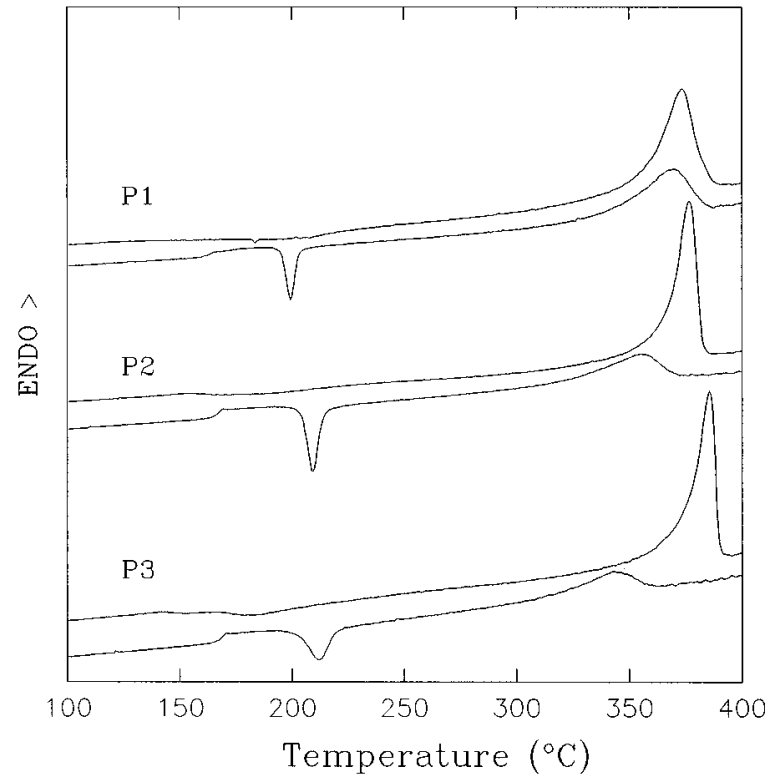

Figure 1. First (top) and second (bottom) DSC heating scans for the three different aromatic polyketones.

\begin{tabular}{|c|c|c|c|c|c|c|c|}
\hline \multirow[b]{2}{*}{ sample } & \multicolumn{2}{|c|}{ first DSC scan } & \multicolumn{5}{|c|}{ second DSC scan } \\
\hline & $\mathrm{T}_{\mathrm{m}}\left({ }^{\circ} \mathrm{C}\right)$ & $\begin{array}{l}\Delta \mathrm{H} \\
(\mathrm{J} / \mathrm{g})\end{array}$ & $\mathrm{T}_{\mathrm{g}}\left({ }^{\circ} \mathrm{C}\right)$ & $\mathrm{T}_{\mathrm{c}}\left({ }^{\circ} \mathrm{C}\right)$ & $\begin{array}{c}\Delta \mathrm{H}_{\mathrm{c}} \\
(\mathrm{J} / \mathrm{g})\end{array}$ & $\mathrm{T}_{\mathrm{m}}\left({ }^{\circ} \mathrm{C}\right)$ & $\begin{array}{c}\Delta \mathrm{H} \\
(\mathrm{J} / \mathrm{g})\end{array}$ \\
\hline $\begin{array}{l}\text { P1 } \\
\text { P2 } \\
\text { P3 }\end{array}$ & $\begin{array}{l}373.5 \\
376.6 \\
385.6\end{array}$ & & $\begin{array}{l}166.5 \\
168.0\end{array}$ & & $\begin{array}{r}-9.7 \\
-16.8 \\
-15.4\end{array}$ & & $\begin{array}{l}31.7 \\
23.4 \\
15.8\end{array}$ \\
\hline
\end{tabular}

particle size was taken as the reference axis when irradiated with polarized light.

\section{Results}

Thermal Behavior. Figure 1 shows the first DSC traces from polymers and the consecutive second scans after a rapid cooling $(320 \mathrm{~K} / \mathrm{min})$ of the sample to 90 ${ }^{\circ} \mathrm{C}$. The glass transition and melting temperatures and the enthal pies of fusion are shown in Table2. F or these three polymers the first DSC runs show a small, very broad exotherm $\left(150-180^{\circ} \mathrm{C}\right)$ and melting points at 374 , 377 , and $386{ }^{\circ} \mathrm{C}$, respectively. The small broad exotherms are associated with some reorganization processes upon heating the material. The melting point and the heat of fusion of the polyketones increase with increasing molecular weight. The second DSC scans show a well-defined glass transition followed by a rather sharp cold crystallization peak and finally by a broad melting peak. The glass transition, $T_{g}$, and the crystallization peak, $T_{c}$, values increase with the molecular weight. However, the melting temperature, $T_{m}$, of the polymers from the second scan decreases with increasing molecular weight. Enthal pies of melting from the second scan were found to decrease in the same order.

Figure 2 represents the first DSC run for sample P3 before and after annealing at the temperatures $\mathrm{T}_{\mathrm{a}}=$ 320 and $345^{\circ} \mathrm{C}$ for $40 \mathrm{~min}$. The large sharp endothermic peak remains essentially constant and a small endothermic peak appears at about 15 deg above the $\mathrm{T}_{\mathrm{a}}$ value used, in agreement with observations for the double melting behavior. ${ }^{4-6}$ The DSC traces for the annealed material did not show any exothermic area. 


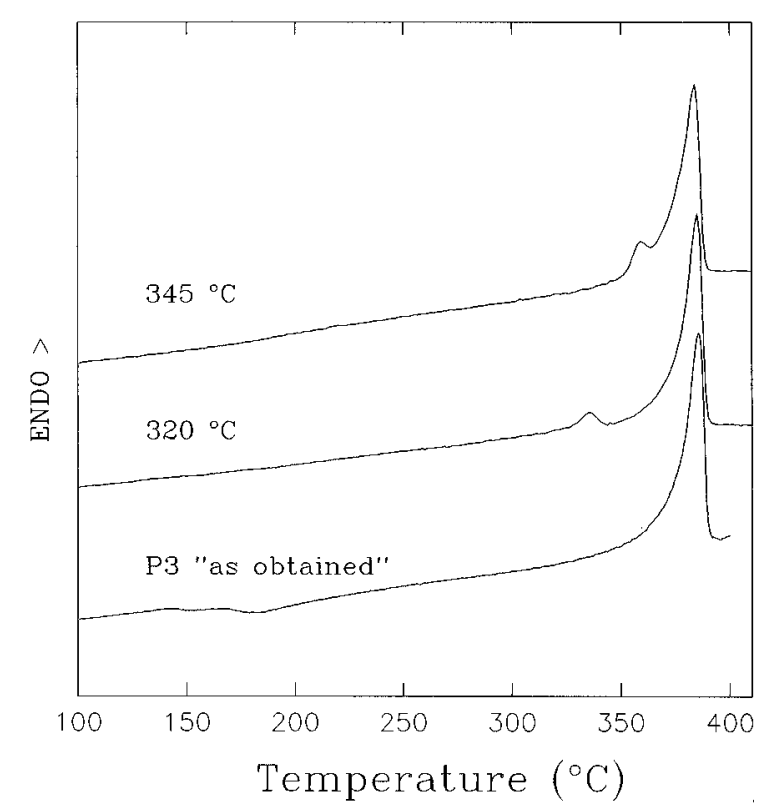

Figure 2. First DSC traces for the sample $\mathrm{P} 3$ before and after annealing at 320 and $345{ }^{\circ} \mathrm{C}$ for $0.7 \mathrm{~h}$.

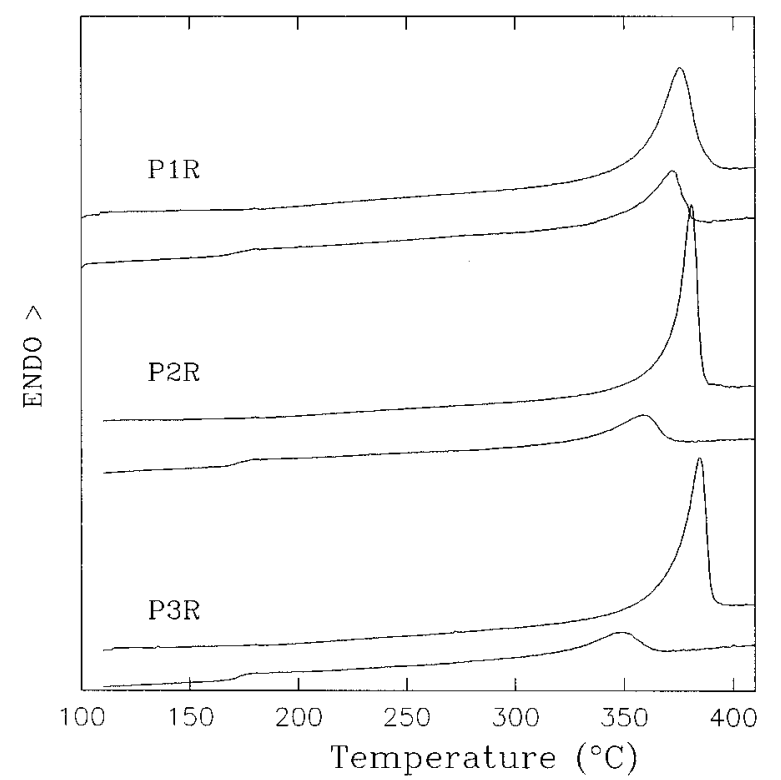

Figure 3. Same as Figure 1 but relative to the reprecipitated samples.

The as obtained polyketones consist of particles widely differing in shape and size (see Experimental Section). Therefore, reprecipitation of the polyketones from solution should erase any architectural features of the particles, thus allowing us to carry out a comparison of the physical properties of the three polymer samples under the same conditions. Figure 3 shows the first and second DSC traces of the reprecipitated samples as described in the experimental part. Their characteristic thermal properties measured are presented in Table 3. The first DSC runs show endothermic peaks similar to those of the original samples (see Figure 1 and Table 3). However, the exothermic area around $180^{\circ} \mathrm{C}$ is much less pronounced than for the original samples. Concerning the second DSC runs, these do not exhibit any cold crystallization peak. The $T_{g}$ and $T_{m}$ values observed in the second DSC run for reprecipitated samples are systematically higher than those observed for the original samples (see Tables 2 and 3). Furthermore, a small increase of the fusion a
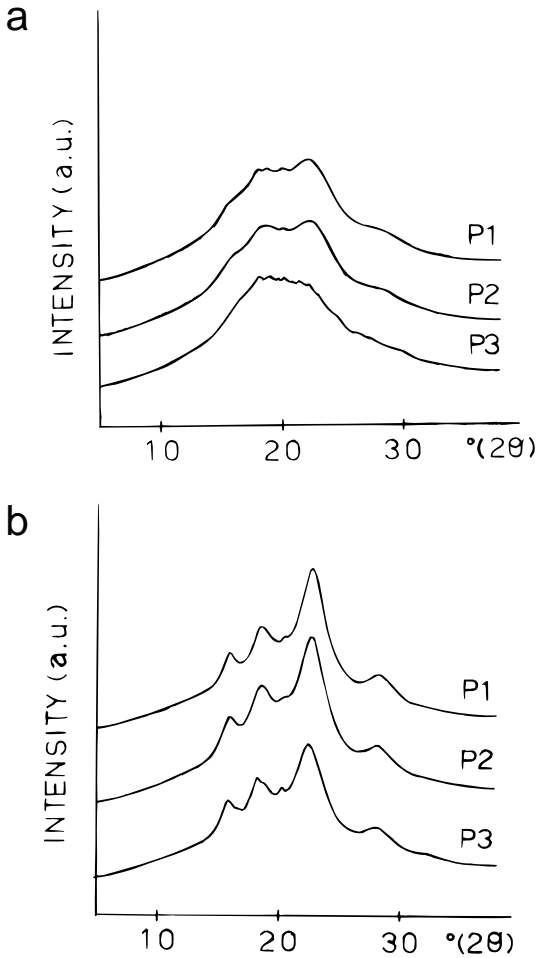

Figure 4. WAXD diffractograms for sintered polyketones samples before (a) and after (b) annealing at $230{ }^{\circ} \mathrm{C}$ for 15 min.

Table 3. Thermal Properties from the First and Second DSC Runs of "Reprecipitated" Polyketones Samples

\begin{tabular}{ccccccc}
\hline & \multicolumn{2}{c}{ first DSC scan } & & \multicolumn{3}{c}{ second DSC scan } \\
\cline { 2 - 3 } \cline { 5 - 7 } sample & $\mathrm{T}_{\mathrm{m}}\left({ }^{\circ} \mathrm{C}\right)$ & $\Delta \mathrm{H}(\mathrm{J} / \mathrm{g})$ & & $\mathrm{T}_{\mathrm{g}}\left({ }^{\circ} \mathrm{C}\right)$ & $\mathrm{T}_{\mathrm{m}}\left({ }^{\circ} \mathrm{C}\right)$ & $\Delta \mathrm{H}(\mathrm{J} / \mathrm{g})$ \\
\hline $\mathrm{P} 1$ & 375.6 & 64.6 & & 168.3 & 372.0 & 32.2 \\
$\mathrm{P} 2$ & 380.9 & 64.3 & & 172.0 & 358.2 & 25.1 \\
$\mathrm{P} 3$ & 384.7 & 64.5 & & 171.6 & 349.0 & 20.3
\end{tabular}

enthalpy values was observed for the reprecipitated samples in relation to the as obtained ones.

X-ray Diffraction. Figure 4a illustrates the wide angleX-ray diffractograms from sintered samples of the three polymer particles as obtained. All three samples show poorly defined scattering patterns. P1 and P2 samples exhibit broad and weak diffraction peaks with the maximun intensity around $23^{\circ}(2 \theta)$. In the case of P3 the diffractogram shows a different intensity distribution. After a thermal treatment at $230{ }^{\circ} \mathrm{C}$ for $15 \mathrm{~min}$ the sintered samples showed an improvement in the definition of the crystal peaks and the degree of crystallinity (Figure $4 b$ ). The diffractograms of the three samples are similar and can be considered equivalent to the scattering patterns reported for pol yketones with a high carbonyl content (PEKK and PEKEKK) prepared under cold crystallization or from solvent crystallization; i.e. they exhibit the polymorphic form II. The reflection at $\sim 15.6^{\circ}$ (indexed as 010) appears well isolated and it allows a quick recognition of the crystallographic form II. In the case of P3 the presence of other low-intensity reflections at about 18 and $20^{\circ}(2 \theta)$ points to the coexistence of both crystallographic forms (I and II).

Parts $a$ and $b$ of Figure 5 show the X-ray diffractograms corresponding to the sintered reprecipitated samples before and after annealing at $230^{\circ} \mathrm{C}$ for $15 \mathrm{~min}$. These diffractograms correspond to the polymorphic form I, which shows the strongest reflection at $\sim 18^{\circ}$ (indexed as 110).

Further annealing of P3 at higher temperatures provokes important changes in the relative intensity of 
a

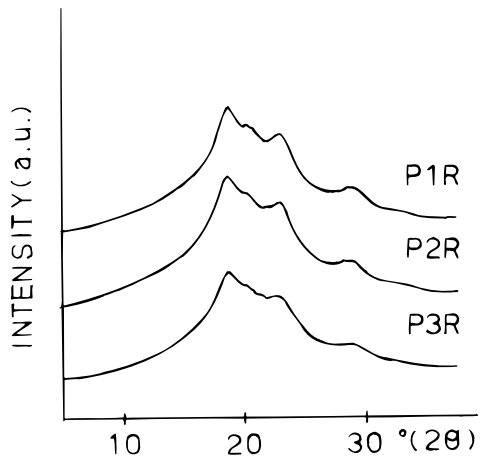

b

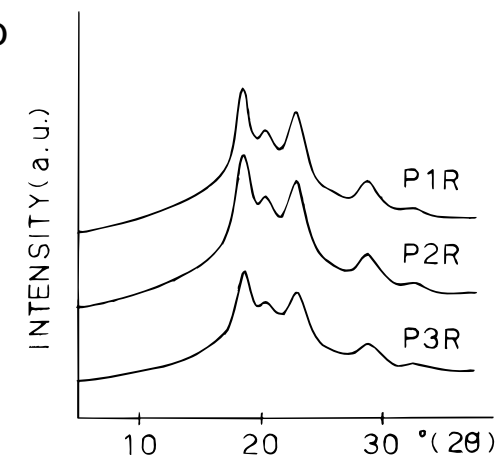

Figure 5. WAXD diffractograms for sintered, reprecipitated samples: before (a) and after (b) annealing at $230{ }^{\circ} \mathrm{C}$ for 15 $\min$.

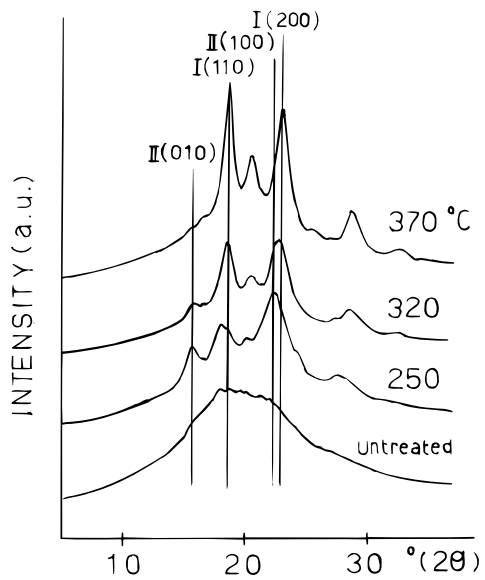

Figure 6. WAXD diffractograms for sintered sample P3 untreated and after consecutive annealing at 250,320, and $370{ }^{\circ} \mathrm{C}$ for $15 \mathrm{~min}$.

Table 4. X-ray Crystallinity in Percent of "As Obtained" and "Reprecipitated" PEKEKK Samples before and after Annealing at $230{ }^{\circ} \mathrm{C}$ for $15 \mathrm{~min}$

\begin{tabular}{cccccc}
\hline & \multicolumn{2}{c}{ as obtained } & & \multicolumn{2}{c}{ reprecipitated } \\
\cline { 2 - 3 } \cline { 5 - 6 } sample & untreated & annealed & & untreated & annealed \\
\hline P1 & 11 & 26 & & 17 & 25 \\
P2 & 13 & 27 & & 15 & 24 \\
P3 & 9 & 24 & & 13 & 23
\end{tabular}

the diffraction peaks (Figure 6). Thus, with increasing temperature, an intensity decrease of the peaks corresponding to form II and a concurrent intensity increase in the maxima associated with form I is observed. Form II disappears at higher temperatures $\left(375^{\circ} \mathrm{C}\right)$.

The X-ray crystallinity values from the as obtained and reprecipitated samples before and after annealing are presented in Table 4. The crystallinity values for the sintered samples are smaller than for the reprecipitated ones. After annealing, both the as obtained

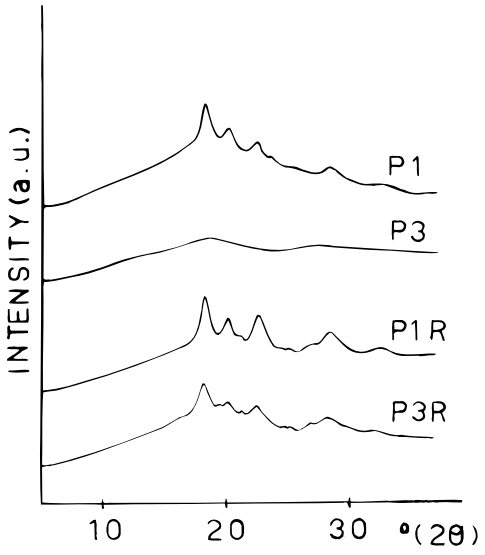

Figure 7. WAXD diffractograms for samples $\mathrm{P} 1$ and $\mathrm{P} 3$ after the second DSC run for samples before (top) and after (bottom) reprecipitation.

and the reprecipitated samples show similar crystallinity values. For the reprecipitated samples the degree of crystallinity appears to decrease with increasing viscosity or $\mathrm{M}_{\mathrm{w}}$.

X-ray diffractograms were al so obtained for the meltcrystallized material (samples after second DSC run). Samples as obtained showed much less defined diffractograms than the reprecipitated samples (Figure 7). Thus, while sample P1 shows a diffractogram similar to that of P1R, sample P2 shows a lower crystallinity (not included in Figure 7) and P3 can be considered amorphous.

Although sample P 3 shows a large number of isolated, very small needl elike particles together with the larger ones, a separation of the needlelike particles, in a convenient amount, from the larger particles was unsuccessful. Glass capillaries with 0.7 and $2 \mathrm{~mm}$ diameters were filled with material from the P 3 sample and theX-ray scattering patterns were obtained. While the thin capillary sample only showed a very broad ring centered around $21^{\circ}(2 \theta)$, the wider one showed an X-ray pattern with well-defined rings. These results suggest that the smallest particles of P3 are mainly amorphous.

Infrared Dichroism Study. In the case of sample P3 the optical and SEM photographs reveal the presence of very small needlelike particles which show welldefined crystal habits. Nevertheless, as mentioned above, the X-ray scattering patterns of these particles reveal the occurrence of an amorphous structure. Since the appearance of crystal habits in a particle would be the result of some molecular organization during its growth, we have used infrared dichroism ${ }^{16}$ for the investigation of these needlelike particles.

Figure 8 illustrates the polarized infrared spectra of a single needle particle of the P3 sample. The longest particle size direction was taken as the reference to apply parallel and perpendicular polarized light. We can see that those bands which can be related to the phenylene ring ${ }^{17}$ (1589 and $1495 \mathrm{~cm}^{-1}\left(v_{\mathrm{C}-\mathrm{C}}\right), 1162$ and $927 \mathrm{~cm}^{-1}$ (out of plane $\mathrm{H}$ vibration mode in the paraphenylene ring)) and the set of bands (1309, 1276 and $1235 \mathrm{~cm}^{-1}$ ) associated with the phenylene linked to the keto grou ${ }^{18}$ and aromatic ether group show a remarkable dichroism. The intensity of these bands is much larger for parallel polarized light. On the contrary, the band at $1657 \mathrm{~cm}^{-1}$, associated with the keto group ${ }^{17}$ shows a very small dichroism and the band intensity is slightly larger for the perpendicular polarization. 


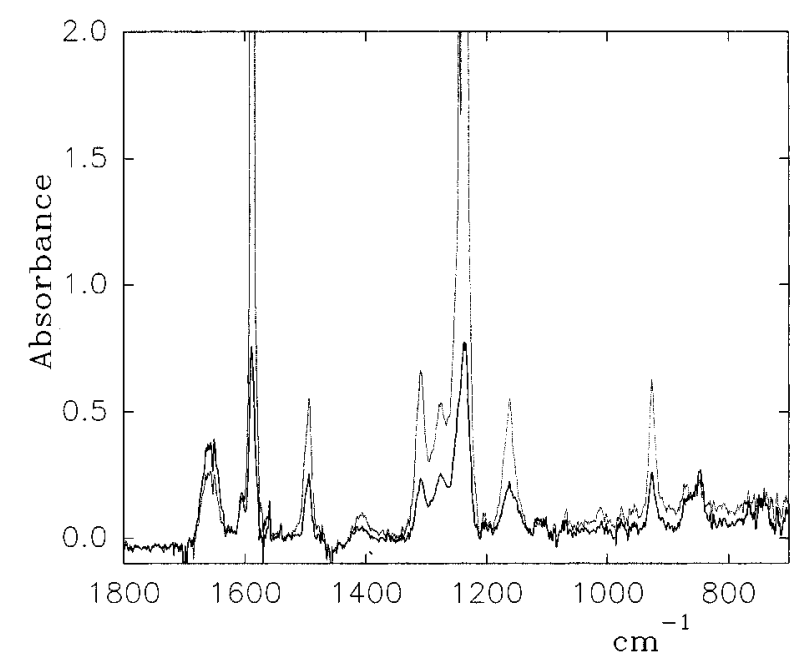

Figure 8. Polarized infrared spectra of a single needlelike particle of P3 sample: (dotted line) II; (solid line) $\perp$.

\section{Discussion}

The above results will be discussed with reference to the influence of polymerization conditions on the thermal properties and to the microstructure of the particles as obtained and of their modification after annealing and reprecipitation. The influence of molecular weight (viscosity) and isomeric defect structures on the architecture of particles as obtained and their thermal properties will also be pointed out.

(a) Thermal Properties. The DSC thermograms of the obtained polyketones reveal the melting behavior which is typical of semicrystalline polymers. It is to be noted that the thermal properties measured from both the first and second heating DSC scans seem to depend mainly on molecular weight (Table 2). Hence, an increase of molecular weight (viscosity) results in an increase of the melting points observed in the first DSC scan. Furthermore, a broader endothermic peak is observed for sample P1 which shows the smallest viscosity value but the highest amount of defect structures (Table 1). Thus, a contribution of defect structures to the observed lowering of $T_{m}$ (from P1 to P3) cannot be discarded. According to the second DSC runs the $\mathrm{T}_{\mathrm{g}}$ values increase with molecular weight as expected. However, the $T_{m}$ values decrease with increasing molecular weight. This could indicate a decreasing size or quality of the crystals grown on cooling the sample after melting. Such an effect could be due to a decreasing chain mobility going from P1 to P3 because of the differences observed in molecular weight.

It is worthwhile to compare the thermal behavior observed for samples as obtained (Figure 1 and Table 2) with that of the reprecipitated samples (Figure 3 and Table 3). As illustrated el sewhere, ${ }^{15}$ the samples show very different morphologies when observed by optical microscopy.

By reprecipitation of the samples we are erasing the likely influence of the particles architecture on the thermal properties which will reflect, in turn, the influence of molecular weight and microstructure of the sample. Since weight losses during repreci pitations are small (less than 2.5\%), the chemical composition of the reprecipitated polyketones should be assumed to be identical to that of the obtained polyketones. On the whole, a systematic increase of $T_{m}$ and $T_{g}$ and enthal pic values was found for the reprecipitated samples in relation to the as obtained ones. Besides the changes observed in $\mathrm{T}_{\mathrm{m}}$ and $\mathrm{Tg}$ values after reprecipitation of
Table 5. Influence of Sample Preparation on the Polymorphism in PEKEKK Materials

\begin{tabular}{ccclc}
\hline & & & \multicolumn{2}{c}{ melt crystallized } \\
\cline { 4 - 4 } & as obtained & reprecipitated & as obtained & reprecipitated \\
\hline P1 & form II $+(\text { form I })^{a}$ & form I & form I & form I \\
P2 & form II $+(\text { form I })^{a}$ & form I & form Ic & form I \\
P3 form II + form Ib & form I & amorphous & form I
\end{tabular}

a Form I could not be ruled out. ${ }^{b}$ Form II is dominant. c Sample with very low crystallinity.

the samples (see Tables 2 and 3) the corresponding second DSC runs reveal a clear different thermal behavior between as obtained and reprecipitated samples. In the first case, a cold crystallization peak is observed for the three samples, while in the case of reprecipitated samples, the cold crystallization peak is not observed. This result could be attributed to a different ability of the samples to crystallize on cooling after the first DSC run. Hence, the capability for crystallization is total in the case of reprecipitated samples and only partial for as obtained samples. Thus, the cold crystallization enthal py values are smaller than the melting enthal pies for P1 and P2 samples (Table 2) while for P3 these values are similar. The difference between both crystallization and mel ting enthal pies is much larger for $\mathrm{P} 1$ (22 J $/ \mathrm{g}$ ) than for P2 $(6.6 \mathrm{~J} / \mathrm{g}$ ) and nil for P3.

(b) Polymorphism and Crystallization Conditions. The variations observed in the X-ray diffractograms (Figure 6) and diffraction patterns upon annealing of the samples are in line with the polymorphism observed in related polyketones. We can say that the as obtained samples appear predominantly in the crystallographic form II, though the presence of form I is not ruled out. Upon annealing, a crystal transformation of form II into form I occurs. This crystal conversion seems to be more effective for higher annealing temperatures and also for longer thermal treatments, as expected. It is worth noting that polyketones as obtained, i.e. crystallized from solution reaction (precipitation polycondensation), exhibit mainly form II (see Figure 4) while the reprecipitated samples show only form I (Figure 5).

The X-ray diffractograms obtained from the meltcrystallized samples (from DSC measurements after the second run) clearly support the above mentioned differences between reprecipitated and as obtained samples (Figure 7). In the case of samples as obtained only sample $P 1$ shows $X$-ray scattering patterns similar to those of reprecipitated samples while the diffractogram of sample P2 (not shown in the figure) denotes a very low crystallinity level and that of sample P3 would correspond to an amorphous material. In the case of reprecipitated samples the X-ray diffractograms reveal a similar semicrystalline character for all three samples. It is noteworthy that the X-ray diffractograms of meltcrystallized material correspond to form I, in agreement with Gardner's observations. Table 5 summarizes the influence of the preparation conditions on the polymorphism observed in these polyketone materials containing a proportion of carbonyl linkage groups of $60 \%$.

(c) Arrangement of the Polyketone Chains in the Needlelike Particles. It is worthwhile to recall the amorphous character exhibited by the smallest particles of sample P3 (which showed birefrigence and crystal habit), while larger P3 particles are semicrystalline. A short thermal treatement of the sample is sufficient to develop crystallinity in these particles. This indicates that for this particular sample having a very 
much higher viscosity (molecular weight) the still growing chains in the precipitated particles could not pack properly (i.e. in crystallographic register), yielding both amorphous and poorly semicrystalline material. However, results obtained from IR dichroism indicate that polymer chains are aligned parallel to the longest dimension of the needle particle. Taking into account the chain conformation of linear polyketones in which ether and keto groups, linking para-phenylene rings, are on the edges of an all-trans planar conformation, ${ }^{7-10}$ the infrared results shown in Figure 8 can be explained if polyketone chains lie parallel al ong the longest particle dimension. Furthermore, the chains would laterally pack at random which would result in a uniaxial orientation with the main axis parallel to the direction of the longest particle size. As a result the $\mathrm{C}-\mathrm{C}$ and $\mathrm{C}-\mathrm{H}$ bonds of the phenylene rings would show a permanent dipolar moment parallel to the chain direction and, consequently, their associated IR bands will exhibit dichroism. On the other hand, since the stretching vibration mode of the $\mathrm{C}=\mathrm{O}$ bond is perpendicular to the chain direction and because of uniaxial orientation of needle particles, the corresponding band at 1657 $\mathrm{cm}^{-1}$ will not exhibit such a high dichroism. Indeed, only a small dichroism is observed for the $v_{\text {CO }}$ band at $1657 \mathrm{~cm}^{-1}$.

(d) Isomeric Chain Defects and Chain Entanglements. From the foregoing the influence of molecular weight on the chain mobility is evident. Chain mobility is, in turn, responsible for the thermal behavior observed and it determines the ability of chains to crystallize from the melt. However, even if the relative amount of defect structures is small (Table 1), their influence on the architecture developed in the growing precipitated particles cannot be discarded. One may speculate that the presence of defect structures (ortho and meta) might be responsible for an entanglement between growing chain segments. Chain entanglements could be released by reprecipitation of the samples, allowing the material to crystallize better, as indicated by the higher enthal py and $T_{m}$ values observed in relation to those for as obtained samples.

Taking into account the relatively high viscosity values observed for the three samples (Table 1) and given the low flexibility of polyketone chains one could attribute the increasing $T_{m}$ values not exclusively to the changes in $\mathrm{M}_{\mathrm{w}}$ (viscosity) but also to the smaller amount of defect structures found when going from P1 to P3.

One might think that reprecipitated polymers could crystallize from the melt more easily than as obtained ones do, as occurs here. One may speculate that this could be due to a chain disentanglement during polymer solution which would increase the chain mobility in the reprecipitated polymers in relation to that of as obtained polyketones.

\section{Conclusions}

The level of crystallinity and the melting behavior of the polyketones investigated primarily depends on the molecular weight. Similar to other polyketones, PEKEKK shows two polymorphic forms: form II, less stable, is currently observed in the samples, while the reprecipitated polymer preferentially shows form I. Form II is partially converted into form I upon short annealing treatment of the material, and it is totally converted after crystallization from the melt. The crystallinity of samples seems to decrease with increasing molecular weight (viscosity), while the corresponding melting temperatures increases. Second DSC runs show decreasing $T_{m}$ values with increasing molecular weight in both as obtained and reprecipitated samples. This result highlights the influence of molecular weight on the chain mobility and consequently on the crystallization process from the melt as well as the thermal behavior observed upon heating. Similarly to molecular weight, isomeric defects in the chains also seem to govern the building up of the particles, thus affecting the chain mobility which finally determines the thermal behavior and the ability of chains to crystallize.

Acknowledgment. Grateful acknowledgment is due to DGICYT, Spain, for the support of this investigation (grant PB94-0049). M.G.Z. also thanks DGICYT for the tenure of a sabbatical grant during his research work in Madrid.

\section{References and Notes}

(1) Staniland, P. A. In Comprehensive Polymer Science; Allen, G., Bevington, J . C., Eds.; Pergamon: Oxford, U.K., 1989; Vol. 5, pp 483-497.

(2) Millins, M. J .; Woo, E. P. J . Macromol. Sci.-Rev. Macromol. Chem. Phys. 1987, C27, 313.

(3) Handbook of polymer synthesis, Part A; Kricheldorf, H. R., Eds.; Marcel Dekker: New York, 1992; p 545.

(4) Blundell, D. J .; Osborn, R. N. Polymer 1983, 24, 953.

(5) Cheng, S. Z. D.; Cao, M. Y.; Wunderlich, B. Macromolecules 1986, 19, 1868.

(6) Bassett, D. C.; Olley, R. H.; Al Raheil, I. A. M. Polymer 1988, 29, 1745

(7) Boom, J .; Magre, E. P. Makromol. Chem. 1969, 126, 130.

(8) Dawson, P. C.; Blundell, D. J . Polymer 1980, 21, 577.

(9) Rueda, D. R.; Ania, F.; Richarson, A.; Ward, I. M.; BaltáCalleja, F. J. Polym. Commun. 1983, 24, 258.

(10) Wakelyn, N. T. Polym. Commun. 1984, 25, 306.

(11) Blundell, D. J .; Newton, A. B. Polymer 1991, 32, 308.

(12) Gardner, K. H.; Hsiao, B. S.; Matheson, R. R., J r.; Wood, B. A. Polymer 1992, 33, 2483.

(13) Gardner, K. H.; Hsiao, B. S.; Faron, K. L. Polymer 1994, 35, 2290.

(14) Cheng, S. Z. D.; Ho, R. M.; Hsiao, B. H.; Gardner, K. H. Macromol. Chem. Phys. 1996, 197, 185.

(15) Zolotukhin, Z.; Rueda, D. R.; Bruix, M.; Cagiao, M. E.; BaltáCalleja, F. J .; Bulai, A.; Gileva, N. G. Submitted for publication to Macromol. Chem. Phys.

(16) Zbinden, R. Infrared Spectroscopy of High Polymers; Academic Press: New York, London, 1964.

(17) Bellamy, L. J . The infrared Spectra of Complex Molecules, 3rd ed.; Chapman and Hall: London, 1979.

(18) Colthup, N. B. J . Opt. Soc. Am. 1952, 40 (6), 397.

MA960482S 